\title{
Influence of chemical stresses in the permeation, one-side and two-side charging processes
}

\author{
Sun-Chien Ko \\ Telecommunication Laboratories, Chung Hua Telecom Company, Taoyuan, Taiwan \\ Tong-Yi Zhang \\ Department of Mechanical Engineering, Hong Kong University of Science and Technology, \\ Clear Water Bay, Kowloon, Hong Kong, China \\ Sanboh Lee ${ }^{\text {a) }}$ \\ Department of Materials Science and Engineering, National Tsing Hua University, Hsinchu, Taiwan
}

(Received 3 February 2007; accepted 31 March 2007; published online 8 June 2007)

\begin{abstract}
Chemical stresses induced during a diffusion process have a great influence on the diffusion process by enhancing the diffusion coefficient. The effective diffusion coefficient is a function of the concentration when chemical stresses are taken into account. The diffusion equation with the effective diffusion coefficient is numerically solved for the three cases, namely, permeation through a membrane, one-side charging and two-side charging of membranes, with the flux continuity boundary conditions, which include the surface effect, i.e., the adsorption, absorption and desorption reactions. The results indicate that chemical stresses enhance the diffusion process. If chemical stresses are neglected, both two-side charging and one-side charging have the same concentration profiles. However, when the chemical stresses are fed back to the diffusion, the concentration level for the two-side charging is higher than that for the one-side charging. The surface effect is also investigated by changing the ratio of the drift velocity in surface to the drift velocity in bulk, showing that the diffusion process is promoted by the increase of the drift velocity ratio. (C) 2007 American Institute of Physics. [DOI: 10.1063/1.2737383]
\end{abstract}

\section{INTRODUCTION}

The permeation test is a common experimental method to measure the diffusion coefficient of a diffusive species in a host material if the concentration distribution of the diffusive species is difficultly detected. Daynes ${ }^{1}$ proposed a timelag method to determine the diffusion coefficient based on the solution of Fick's second law with the constant concentration boundary conditions at the entry and exit surfaces. The approach with constant concentration boundary conditions may be called the constant concentration model for simplicity. The time-lag method may lead to a large deviation of the determined diffusion coefficient if the surface effect and traps to diffusive species play predominant roles in the permeation process. For example, Völkl and Alefeld ${ }^{2}$ summarized 46 sets of data for the hydrogen permeation in $\alpha$-iron. They found that the experimental data were scattered in 3-4 orders of magnitude due to the surface effect and hydrogen traps. The surface effect is caused by the absorption and desorption processes, occurring at the entry and exit surfaces during the permeation process, which leads to a thickness-dependent diffusion coefficient when it is determined from the time-lag method. ${ }^{3,4}$ Wang $^{5}$ analyzed the adsorption, absorption, and desorption processes with two forward jumps and two backward jumps of the diffusive species at the entry and exit surfaces based on the permeation flux at steady state. Following Wang's approach, Zhang and Zheng ${ }^{6,7}$ and Zhang, Zheng, and $\mathrm{Wu}^{8}$ proposed the flux con-

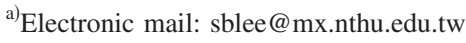

tinuity model, which means that Fick's second law is solved with the flux continuity boundary conditions at the entry and exit surfaces. A desorption rate constant and an absorption parameter are introduced in the flux continuity model to describe the surface effect in the permeation process. The diffusion coefficient evaluated from the flux continuity model is independent of the sample thickness and the surface treatment. In addition, one is able to determine the desorption rate constant and the absorption parameter with the flux continuity model. Both the desorption rate constant and the absorption parameter depend on the surface treatment and may be independent of the sample thickness.

There is another challenging issue in studying diffusion. Chemical stresses are built-up by composition inhomogeneneity during mass transfer. Chemical stresses developed in diffusion are internal stresses, which can damage or improve, depending on the distribution and magnitude of the stresses, the mechanical properties of materials ${ }^{9}$ and electrical properties of semiconductor materials. ${ }^{10}$ When chemical stresses are high enough, they can cause phase transformation, dislocation generation, cracks, etc. Those defects are possible to trap electron and hole in semiconductor devices so that their performance becomes worse. For example, dislocations induced by chemical stresses in originally defect-free silicon crystals were observed by using transmission electron microscopy ${ }^{11}$ and x-ray topography, ${ }^{12}$ after phosphorus atoms were diffused into the crystals.

Prussin ${ }^{13}$ and $\mathrm{Li}^{14}$ are pioneers in the research on chemical stresses during mass transfer. Following their approaches, Lee and co-workers ${ }^{15-18}$ solved various problems of chemi- 
cal stresses with different geometries and boundary conditions. On the other hand, stresses play a certain role in a diffusion process, which has also attracted many researchers. For example, Larche and Cahn ${ }^{19,20}$ investigated the stress dependence of local diffusion flux in a solid. Chemical stresses are generated during diffusion, which, in turn, affect the diffusion process by affecting the diffusion coefficient. In the present work, we derive a diffusion equation based on the thermodynamics by taking chemical stresses into account. Then, three processes of permeation, one-side charging and two-side charging are studied with the flux continuity boundary conditions.

\section{ANALYSIS}

Based on thermodynamic theory, when a mobile solute in an ideal and dilute solid solution is subjected to stresses, its chemical potential, $\mu$, is given by

$$
\mu=\mu_{0}+R T \ln C-\sigma_{h} \bar{V}
$$

where $\mu_{0}$ is a reference chemical potential independent of concentration and stresses, $R$ is the gas constant, $T$ denotes absolute temperature, $C$ and $\bar{V}$ are the concentration and the partial molal volume of the mobile solute, respectively, and $\sigma_{h}$ denotes hydrostatic stress. In the present work, we consider only the case that a stress field will be instantly built-up for a given concentration distribution. Thus, during a diffusion process through a membrane of a thickness $L$, a solute concentration distribution, $C(x, t)$, along the thickness direction, i.e., the $x$ axis, generates transverse stresses, which are approximately expressed as ${ }^{14}$

$$
\begin{aligned}
\sigma_{y y}=\sigma_{z z}= & -\frac{\bar{V} E}{3(1-\nu)}\left[-C+\frac{1}{L} \int_{0}^{L} C d x\right. \\
& \left.+\frac{6(2 x-L)}{L^{3}} \int_{0}^{L} C\left(x-\frac{L}{2}\right) d x\right],
\end{aligned}
$$

in which $E$ and $\nu$ are Young's modulus and Poisson's ratio of the membrane material, respectively. The second and third terms on the right side of Eq. (2) are due to force balance and moment balance, respectively. Note that the chemical stress component, $\sigma_{x x}$, is zero. Therefore, substituting Eq. (2) into Eq. (1), one obtains

$$
\begin{aligned}
\mu= & \mu_{0}+R T \ln C-\frac{2 \bar{V}^{2} E}{9(1-\nu)}\left[-C+\frac{1}{L} \int_{0}^{L} C d x\right. \\
& \left.+\frac{6(2 x-L)}{L^{3}} \int_{0}^{L} C\left(x-\frac{L}{2}\right) d x\right] .
\end{aligned}
$$

The diffusion flux is proportional to the chemical potential gradient

$$
J=-M C \frac{\partial \mu}{\partial x}
$$

where $M$ is the solute mobility. Substituting Eq. (3) into Eq. (4) yields

$$
\begin{aligned}
J= & -M R T\left\{1+\frac{2 \bar{V}^{2} E C}{9(1-\nu) R T}\left[1-\frac{12}{L^{3} \partial C / \partial x}\right.\right. \\
& \left.\left.\times \int_{0}^{L} C\left(x-\frac{L}{2}\right) d x\right]\right\} \frac{\partial C}{\partial x} .
\end{aligned}
$$

On the other hand, Fick's first law states

$$
J=-D_{\text {eff }} \partial C / \partial x .
$$

Comparing Eq. (5) with Eq. (6), the effective diffusion coefficient is defined by

$$
D_{\text {eff }}=D_{0}\left\{1+F C\left[1-\frac{12}{L^{3} \partial C / \partial x} \int_{0}^{L}\left(x-\frac{L}{2}\right) C d x\right]\right\},
$$

where

$$
F=\frac{2 \bar{V}^{2} E}{9(1-\nu) R T}
$$

and

$$
D_{0}=M R T
$$

are the diffusion coefficients of the solute without considering the chemical stresses. As indicated by Eq. (8a), the parameter $F$ is proportional to the square of $\bar{V}$ and therefore is always positive. Then, Fick's second law takes the form

$$
\frac{\partial}{\partial x}\left(D_{\mathrm{eff}} \frac{\partial C}{\partial x}\right)=\frac{\partial C}{\partial t} .
$$

Substituting Eq. (7) into Eq. (9) yields

$$
\begin{aligned}
& D_{0}(1+F C) \frac{\partial^{2} C}{\partial x^{2}}+D_{0} F\left(\frac{\partial C}{\partial x}\right)^{2}+\frac{6 D_{0} F}{L^{3}} \frac{\partial C}{\partial x} \int_{0}^{L} C(L-2 x) d x \\
& \quad=\frac{\partial C}{\partial t}
\end{aligned}
$$

Equation (10) will be numerically solved for three cases of diffusion, namely the permeation, one-side charging and two-side charging processes.

\section{PERMEATION}

Consider a specimen of thickness $L$ where the entry side and exit side are located at $x=0$ and $x=L$, respectively. Before permeation starts, the specimen is solute-free. The initial and boundary conditions are given by

$C=0$, for $t=0$; and

$$
\left.\begin{array}{l}
\left(D_{\text {eff }} \frac{\partial C}{\partial x}-k C\right)_{x=0}=-k_{P}, \\
\left(D_{\text {eff }} \frac{\partial C}{\partial x}+k C\right)_{x=L}=0,
\end{array}\right\} \text { for } t>0,
$$

where $k_{p}$ and $k$ denote the absorption parameter and the desorption rate constant, respectively. Zhang and Zheng ${ }^{6}$ introduced the drift velocity on surface, $V_{s}=k$, the drift velocity in bulk 


$$
V_{b}=D_{0} / L
$$

and the permeation concentration

$$
C_{0}=\frac{k_{p}}{k}
$$

We introduce dimensionless length $x^{\prime}$, dimensionless concentration $N$, dimensionless time $t^{\prime}$, and dimensionless parameter $F^{\prime}$ as follows:

$$
\begin{aligned}
& N=C / C_{0}, \\
& x^{\prime}=x / L, \\
& t^{\prime}=D_{0} t / L^{2}, \\
& F^{\prime}=F C_{0} .
\end{aligned}
$$

Using the dimensionless parameters, we rewrite Fick's second law and the boundary conditions as

$$
\begin{aligned}
& \left(1+F^{\prime} N\right) \frac{\partial^{2} N}{\partial x^{\prime 2}}+F^{\prime}\left(\frac{\partial N}{\partial x^{\prime}}\right)^{2}+6 F^{\prime} \frac{\partial N}{\partial x^{\prime}} \int_{0}^{1} N\left(1-2 x^{\prime}\right) d x^{\prime} \\
& =\frac{\partial N}{\partial t^{\prime}} \\
& \left(1+F^{\prime} N\right) \frac{\partial N}{\partial x}+6 F^{\prime} \int_{0}^{1}\left(1-2 x^{\prime}\right) N d x^{\prime}-\frac{V_{s}}{V_{b}} N \\
& \quad=-\frac{V_{s}}{V_{b}} \text { at } x^{\prime}=0, \\
& \left(1+F^{\prime} N\right) \frac{\partial N}{\partial x^{\prime}}+6 F^{\prime} \int_{0}^{1}\left(1-2 x^{\prime}\right) N d x^{\prime}+\frac{V_{s}}{V_{b}} N=0 \text { at } x^{\prime}=1
\end{aligned}
$$

Equation (15) with Eq. (16a) and (16b) is numerically solved by using the finite difference method. The space derivative and time derivative are calculated by the central difference and forward difference, respectively. The integral is calculated using Simpson's rule. The dimensionless membrane thickness is divided into 200 equal parts, i.e., $\Delta x^{\prime}$ $=0.005$. The dimensionless time increment is set to be $\Delta t^{\prime}$ $=0.001$.

Figure 1 shows the concentration distributions at different times for $F C_{0}=1.5$ and $F C_{0}=0$ with $V_{s} / V_{b}=1.5$. The concentration near the entry surface for $F C_{0}=1.5$ is less than that for $F C_{0}=0$ and the difference between them increases with increasing time. However, the concentration at the exit surface has a trend opposite to that at the entry surface. The concentrations at the entry surface and the exit surface as a function of time with various values of $V_{s} / V_{b}$ are shown in Fig. 2 by solid and dashed lines, respectively, where $F C_{0}$ $=0.5$. The concentration at the entry surface increases with increasing $V_{s} / V_{b}$. The concentration at the exit surface increases with increasing $V_{s} / V_{b}$ at the short times and then decreases with increasing $V_{s} / V_{b}$ at the long times. This is because the higher the value of $V_{s} / V_{b}$, the less dominant the surface effect, as discussed in detail in Ref. 6. When the

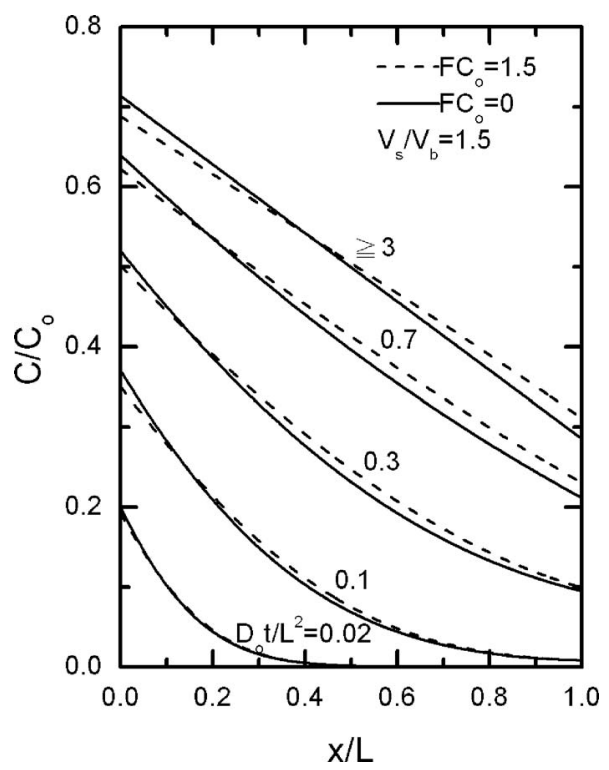

FIG. 1. Concentration profiles at different times for $F C_{0}=0$ and $F C_{0}=1.5$ with $V_{s} / V_{b}=1.5$ in a permeation process.

value of $V_{s} / V_{b}$ approaches infinity, one can reduce the flux continuity model to the constant concentration model. In such a limiting case, the permeation concentration will be reduced to the equilibrium concentration used in the boundary condition at the entry surface in the constant concentration model. The concentration at the exit surface will approach zero when the value of $V_{s} / V_{b}$ approaches infinity. At a given time, the value of $V_{s} / V_{b}$ has a great influence on the concentration distribution, as shown in Fig. 3, where $D_{0} t / L^{2}=0.3$ and $F C_{0}=0.001$. Clearly, a smaller value of $V_{s} / V_{b}$ gives a lower concentration distribution, thereby indicating the significant surface effect in the permeation process.

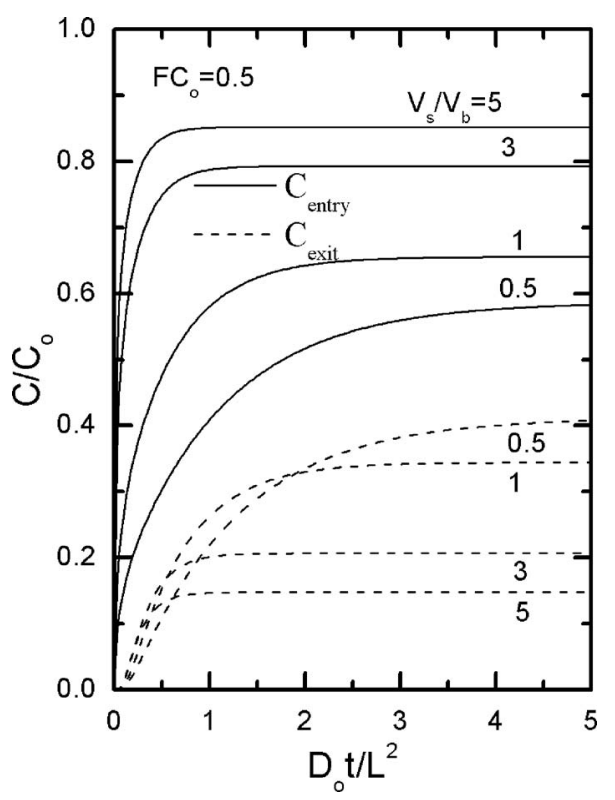

FIG. 2. The solid and dashed lines represent concentrations at the entry surface and the exit surface, respectively, for various values of $V_{s} / V_{b}$ with $F C_{0}=0.5$ in a permeation process. 


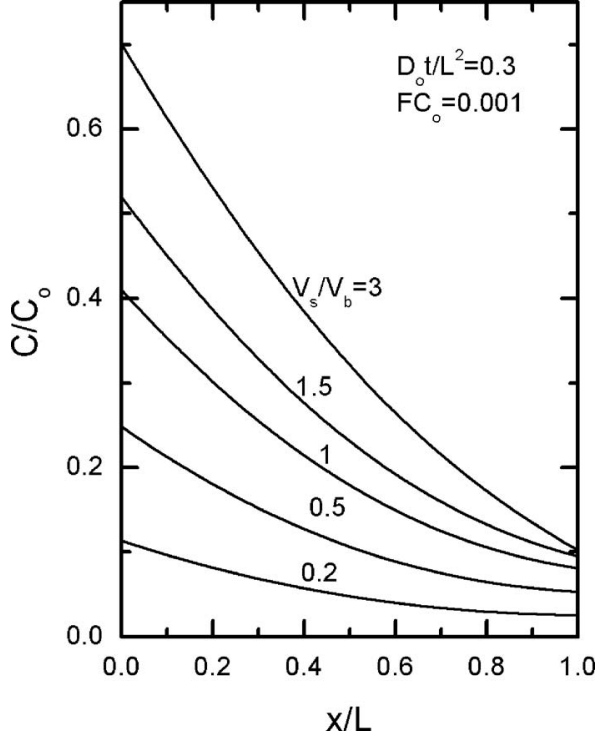

FIG. 3. Concentration profiles for different values of $V_{s} / V_{b}$ with $D_{0} t / L^{2}$ $=0.3$ and $F C_{0}=0.001$ in a permeation process.

\section{ONE-SIDE CHARGING AND TWO-SIDE CHARGING}

Consider a membrane specimen of thickness $L$. Initially no solute is inside the membrane. One-side of the membrane is charged at the surface $x=L$ and the other side, $x=0$, is insulated. The boundary conditions are given by

$$
\begin{aligned}
& \frac{\partial C}{\partial x}=0 \text { at } x=0, \\
& D_{\mathrm{eff}} \frac{\partial C}{\partial x}+k C=k_{p} \text { at } x=L .
\end{aligned}
$$

Equations (17a) and (17b) can be normalized to

$$
\begin{gathered}
\frac{\partial N}{\partial x^{\prime}}=0 \text { at } x^{\prime}=0, \\
\left(1+F^{\prime} N\right) \frac{\partial N}{\partial x^{\prime}}+6 F^{\prime} \int_{0}^{1}\left(1-2 x^{\prime}\right) N d x^{\prime}+\frac{V_{s}}{V_{b}} N=\frac{V_{s}}{V_{b}} \text { at } x^{\prime}=1 .
\end{gathered}
$$

Equation (15) is numerically solved with the initial concentration-free condition and the boundary conditions of Eq. (18a) and (18b). The numerical process is the same as the previous section.

For two-side charging, we let the membrane thickness be $2 L$. Before charging, no solute exists inside the membrane. Solutes are charged from both surfaces and the boundary conditions are given by

$$
\begin{aligned}
& D_{\mathrm{eff}} \frac{\partial C}{\partial x}-k C=-k_{p} \text { at } x=L, \\
& D_{\mathrm{eff}} \frac{\partial C}{\partial x}+k C=k_{p} \text { at } x=L .
\end{aligned}
$$

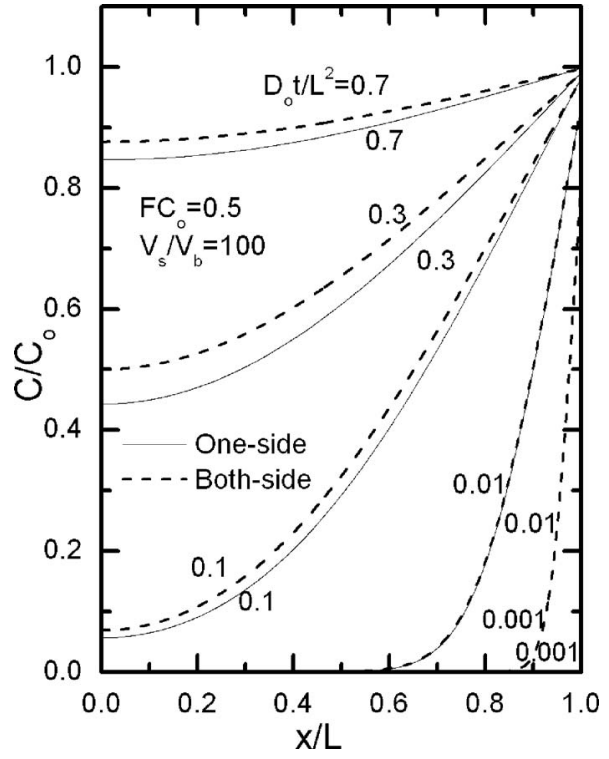

FIG. 4. The solid and dashed lines represent the concentrations at different charging times during one-side charging and two-side charging, respectively, where $F C_{0}=0.5$ and $V_{s} / V_{b}=100$.

Because of geometric symmetry, the concentration is an even function of $x$. Therefore, the effective diffusion coefficient of Eq. (7) is reduced to

$$
D_{\text {eff }}=D_{0}(1+F C)
$$

and the diffusion equation Eq. (9) becomes

$$
D_{0}(1+F C) \frac{\partial^{2} C}{\partial x^{2}}+D_{0} F\left(\frac{\partial C}{\partial x}\right)^{2}=\frac{\partial C}{\partial t} \text {. }
$$

Then, Eqs. (21), (19a), and (19b) are normalized to

$$
\begin{aligned}
& \left(1+F^{\prime} N\right) \frac{\partial^{2} N}{\partial x^{\prime 2}}+F^{\prime}\left(\frac{\partial N}{\partial x^{\prime}}\right)^{2}=\frac{\partial N}{\partial t^{\prime}}, \\
& \left(1+F^{\prime} N\right) \frac{\partial N}{\partial x^{\prime}}-\frac{V_{s}}{V_{b}} N=-\frac{V_{s}}{V_{b}} \text { at } x^{\prime}=-1, \\
& \left(1+F^{\prime} N\right) \frac{\partial N}{\partial x^{\prime}}+\frac{V_{s}}{V_{b}} N=\frac{V_{s}}{V_{b}} \text { at } x^{\prime}=1 .
\end{aligned}
$$

Equation (22) with Eqs. (23a) and (23b) can be solved numerically. Because of the geometric symmetry, the concentration is an even function of $x$. Therefore, the membrane of half thickness, i.e., $0 \leq x^{\prime} \leq 1$, is analyzed. The boundary condition, Eq. (23a), is replaced by

$$
\frac{\partial N}{\partial x^{\prime}}=0 \text { at } x^{\prime}=0 \text {. }
$$

The same numerical process as described earlier is conducted in solving Eq. (22) with the boundary conditions of Eqs. (23b) and (24).

If chemical stresses are neglected, the concentration profile in one-side charging will be identical to the half concentration profile in two-side charging. If chemical stresses are feedback to diffusion, the concentration profile in both processes will be different. Figure 4 shows the concentration 


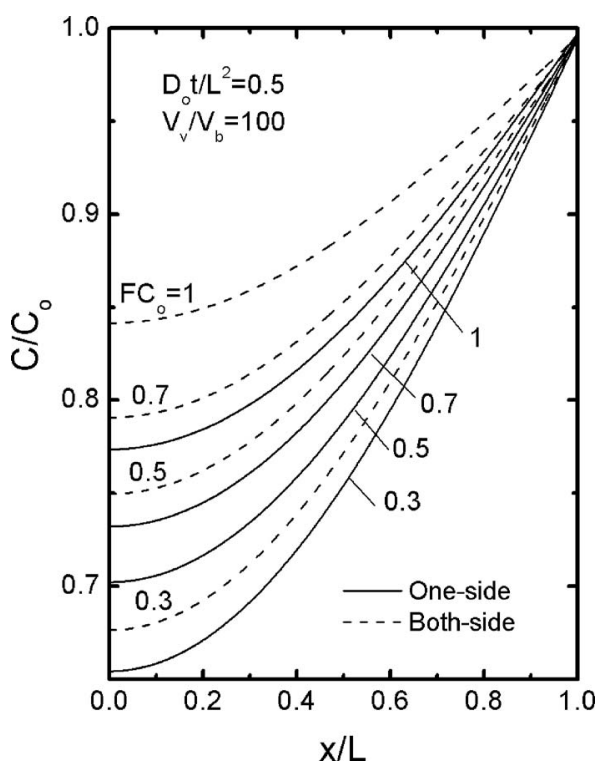

FIG. 5. The solid and dashed lines represent the concentrations for different values of $F$ during one-side charging and two-side charging, respectively, where $D_{0} t / L^{2}=0.5$ and $V_{s} / V_{b}=100$.

profiles at different times for one-side charging and two-side charging, where $F C_{0}=0.5$ and $V_{s} / V_{b}=100$. As expected, more solutes penetrate into the membrane as charging time increases. It is interesting to note that the concentration in one-side charging is less than the concentration in two-side charging and this difference will be maximum at a certain period of charging time, as shown in Fig. 4 for the dimensionless time, $t^{\prime}=0.3$. This is because the chemical stresses induced in the one-side charging are different from that induced in the two-side charging and the difference in the chemical stresses causes the concentration difference. To illustrate the effect of chemical stresses on the charging behavior, we plot the concentration profile for various values of $F C_{0}$ in Fig. 5, where $D_{0} t / L^{2}=0.5$ and $V_{s} / V_{b}=100$. Obviously, the concentration in both cases increases with the value of $F C_{0}$, thereby indicating that the higher the chemical stresses, the higher the concentration. Chemical stresses enhance the diffusion. Figure 6 shows the concentration distribution for various values of $V_{s} / V_{b}$, where $F C_{0}=0.5$ and $D_{0} t / L^{2}=0.2$. Clearly, the concentration level moves up as the value of $V_{s} / V_{b}$ increases. As mentioned earlier, a higher value of $V_{s} / V_{b}$ means the less influence of the surface in the charging process.

\section{DISCUSSION}

According to Eq. (7) and the calculations described earlier, $D_{\text {eff }}$ is always greater than $D_{0}$. The diffusivity enhancement is due to the chemical stresses developed in diffusion and the phenomenon was observed during the diffusion of group III and group V elements in silicon. ${ }^{2 \mathrm{1}}$ For example, the push out effect in a double diffused $n-p-n$ transistor is induced by the fast diffusion of the dopants near the emitter region through dislocations, which are generated by chemical stresses. However, because of complicated phenomenon, no experimental data in the literature can be compared di-

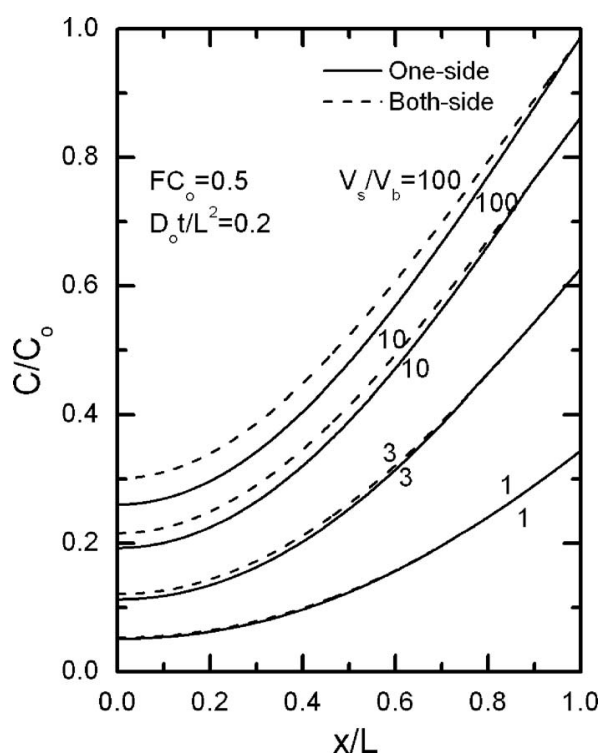

FIG. 6. The solid and dashed lines represent the concentrations at different values of $V_{s} / V_{b}$ during one-side charging and two-side charging, respectively, where $F C_{0}=0.5$ and $D_{0} t / L^{2}=0.2$.

rectly with the theoretical predictions. Well-designed experiments should be performed in the future to verify the theoretical results.

\section{SUMMARY}

Chemical stresses are induced during diffusion, which in turn have great influence on the diffusion process. A selfconsistent diffusion equation including the chemical stresses has been derived from the basic thermodynamic theory and Fick's laws. The chemical stresses enhance the diffusion coefficient and thus promote the diffusion process. If the chemical stresses are negligible, both processes of one-side charging and two-side charging should have the same concentration profile. However, the one-side charging induces the membrane bending due to the asymmetric concentration distribution, while there no bending occurs in the two-side charged membrane. Feedback of chemical stresses makes the concentration level low in the one-side charged membrane than that in the two-side charged membrane.

\section{ACKNOWLEDGMENTS}

This work was supported by the National Science Council of Taiwan. T.Y.Z. was supported by an RGC grant from the Research Grants Council of the Hong Kong Special Administrative Region, China.

${ }^{1}$ H. A. Daynes, Proc. R. Soc. London, Ser. A 97, 286 (1920).

${ }^{2} \mathrm{~J}$. Völhl and G. Alefeld, in Hydrogen in Metals, Topics in Applied Physics, edited by G. Alefeld and J. Völhl (Springer, New York, 1978), Vol. 28, p. 321.

${ }^{3}$ S. Wach, A. P. Miodowink, and J. Mackowiak, Corros. Sci. 6, 271 (1966).

${ }^{4}$ S. Wach and A. P. Miodowink, Corros. Sci. 8, 271 (1968).

${ }^{5}$ J. Wang, Proc. Cambridge Philos. Soc. 32, 657 (1936).

${ }^{6}$ T. Y. Zhang and Y.-P. Zheng, Acta Mater. 46, 5023 (1998).

${ }^{7}$ T. Y. Zhang and Y.-P. Zheng, Acta Mater. 46, 5035 (1998).

${ }^{8}$ T. Y. Zhang, Y.-P. Zheng, and Q. Y. Wu, J. Electrochem. Soc. 146, 1741 (1999).

${ }^{9}$ R. E. Reed-Hill, Physical Metallurgy Principles, 2nd ed. (Van Nostrand, 
New York, 1973), p. 360.

${ }^{10} \mathrm{~B}$. Tuck, Introduction to Diffusion in Semiconductors (Peter Peregrinus, London, 1974), Chap. 8.

${ }^{11}$ M. L. Joshi and J. Wilhelm, J. Electrochem. Soc. 112, 185 (1965).

${ }^{12}$ J. M. Fairfield and G. H. Schwuttke, J. Electrochem. Soc. 115, 415 (1968).

${ }^{13}$ S. Prussin, J. Appl. Phys. 32, 1876 (1961).

${ }^{14}$ J. C. M. Li, Metall. Mater. Trans. A 9A, 1353 (1979).

${ }^{15}$ W. L. Wang, Y. T. Chou, and S. Lee, J. Mater. Res. 16, 1967 (2001).
${ }^{16}$ T. Y. Zhang, S. C. Ko, and S. Lee, J. Appl. Phys. 91, 2002 (2002).

${ }^{17}$ S. C. Ko, S. Lee, and Y. T. Chou, Mater. Sci. Eng., A 409, 145 (2005).

${ }^{18}$ J. L. Chu and S. Lee, J. Appl. Phys. 74, 171 (1993).

${ }^{19}$ F. C. Larche and J. W. Cahn, Acta Metall. 30, 1835 (1982).

${ }^{20}$ F. C. Larche and J. W. Cahn, J. Res. Natl. Bur. Stand. 89, 467 (1984).

${ }^{21}$ W. Frank, U. Gosele, H. Mehrer, and A. Seeger, in Diffusion in Crystalline Solids, edited by G. E. Murch and A. S. Nowick (Academic, New York, 1984), p. 63. 